\title{
FUNDAMENTAL GROUP SCHEMES OF HILBERT SCHEME OF $n$ POINTS ON A SMOOTH PROJECTIVE SURFACE
}

\author{
ARJUN PAUL* AND RONNIE SEBASTIAN
}

\begin{abstract}
Let $k$ be an algebraically closed field of characteristic $p>3$. Let $X$ be an irreducible smooth projective surface over $k$. Fix an integer $n \geq 2$ and let $\mathcal{H} i l b_{X}^{n}$ be the Hilbert scheme parameterizing effective 0 cycles of length $n$ on $X$. The aim of the present article is to find the $S$-fundamental group scheme and Nori's fundamental group scheme of the Hilbert scheme $\mathcal{H} i l b_{X}^{n}$.
\end{abstract}

\section{INTRODUCTION}

Let $X$ be a connected, reduced and complete scheme over a perfect field $k$ and let $x \in X$ be a $k$-rational point. In [Nor76], Nori introduced a $k$-group scheme $\pi^{N}(X, x)$ associated to essentially finite vector bundles on $X$, and in [Nor82], the definition of $\pi^{N}(X, x)$ was extended to connected and reduced $k$-schemes. In [BPS06], Biswas, Parameswaran and Subramanian defined the notion of $S$-fundamental group scheme $\pi^{S}(X, x)$ for $X$ a smooth projective curve over any algebraically closed field $k$. This was generalized to higher dimensional connected smooth projective $k$-schemes and studied extensively by Langer in [Lan11, Lan12]. In general, $\pi^{S}(X, x)$ carries more information than $\pi^{N}(X, x)$ and $\pi^{\text {ét }}(X, x)$. There are natural faithfully flat homomorphisms of affine $k$-group schemes $\pi^{S}(X, x) \rightarrow \pi^{N}(X, x) \rightarrow \pi^{\text {ét }}(X, x)$. The reader is referred to the introductions in [Nor82] and [Lan11] for more details. Precise definitions of the above objects are given in the next section. It is an interesting problem to determine $\pi^{\text {ét }}(X, x), \pi^{N}(X, x)$ and $\pi^{S}(X, x)$ for well-known varieties.

Let $k$ be an algebraically closed field and let $n \geq 2$. Let $\mathcal{H} i l b_{X}^{n}$ be the Hilbert scheme of $n$ points on an irreducible smooth projective surface $X$ over $k$. It is known that $\mathcal{H} i l b_{X}^{n}$ is an irreducible smooth projective variety of dimension $2 n$ over $k$. The geometry of $\mathcal{H} i l b_{X}^{n}$ has been extensively studied, see [Fog73, FGI ${ }^{+}$05, Iar72] and the references therein. In [Bea83] the author computes the topological fundamental group of $\mathcal{H} i l b_{X}^{n}$ when $k=\mathbb{C}$. In

2010 Mathematics Subject Classification. 14J60, 14F35, 14L15, 14 C05.

Key words and phrases. Finite vector bundle, $S$-fundamental group-scheme, Hilbert scheme, semistable bundle, Tannakian category.

*Corresponding author. 
[BH15, Theorem 1.1, Theorem 1.2] Biswas and Hogadi show that the étale fundamental group $\pi^{\text {ét }}\left(\mathcal{H} i l b_{X}^{n}, \widetilde{n x}\right)$ is isomorphic to the abelianization of $\pi^{\text {ét }}(X, x)$, for any $x \in X(k)$. Here $\widetilde{n x}$ is a point in $\mathcal{H} i l b_{X}^{n}$ mapping to $n x \in S^{n}(X)$. Therefore, it is natural to ask if a similar result holds for $\pi^{N}\left(\mathcal{H} i b_{X}^{n}, \widetilde{n x}\right)$ and $\pi^{S}\left(\mathcal{H} i b_{X}^{n}, \widetilde{n x}\right)$. In this paper we answer this question affirmatively when the base field $k$ has characteristic $p>3$. The following is the main result of this article.

Theorem (Theorem 5.3.11). Let $\operatorname{char}(k)>3$ and $n \geq 2$. Then there is an isomorphism of affine $k$-group schemes

$$
\widetilde{f}: \pi^{S}(X, x)_{\mathrm{ab}} \stackrel{\sim}{\longrightarrow} \pi^{S}\left(\mathcal{H} i b_{X}^{n}, \widetilde{n x}\right) .
$$

In particular, $\pi^{S}\left(\mathcal{H} i b_{X}^{n}, \widetilde{n x}\right)$ is an abelian group scheme.

From the above we can easily deduce the following result.

Theorem (Theorem 5.3.12). Let $\operatorname{char}(k)>3$ and $n \geq 2$. Then there is an isomorphism of affine $k$-group schemes

$$
\widetilde{f}^{?}: \pi^{?}(X, x)_{\mathrm{ab}} \stackrel{\sim}{\longrightarrow} \pi^{?}\left(\mathcal{H} i l b_{X}^{n}, \widetilde{n x}\right),
$$

where $?=N$, ét. As above, this shows that the groups $\pi^{?}\left(\mathcal{H} i l b_{X}^{n}, \widetilde{n x}\right)$ are abelian for $?=N$, ét.

The assertion about $\pi^{\text {ét }}\left(\mathcal{H} i l b_{X}^{n}, \widetilde{n x}\right)$ is a corollary of the main result in [BH15], which is proved using a different method.

We briefly describe the organization of this paper. In $\S 2$ we recall the main definitions and results on fundamental group schemes that we need from [Nor82] and [Lan11]. In $\S 3$ we recall and prove results that we need about the Hilbert scheme and the Hilbert-Chow map. The main input in this paper is the construction in $\S 4$, which we briefly explain here. Let $\varphi: \mathcal{H} i l b_{X}^{n} \rightarrow S^{n}(X)$ denote the Hilbert-Chow morphism and let $\psi: X^{n} \rightarrow$ $S^{n}(X)$ denote the quotient map under the natural action of $S_{n}$ on $X^{n}$. Given a numerically flat sheaf $E$ on $\mathcal{H} i l b_{X}^{n}$, we can associate to it a coherent sheaf on $X^{n}$, namely, $\psi^{*} \varphi_{*} E$. However, it is not clear if this coherent sheaf is numerically flat. To remedy this, we associate to $E$ a locally free sheaf on a large open subset of $X^{n}$ and take its unique reflexive extension. Then we use the criterion [Lan12, Theorem 2.2] (this criterion is proved in [Lan11] but stated more precisely in [Lan12]) to check that this reflexive sheaf is locally free. From this construction we are able to define a homomorphism $\pi^{S}(X, x)_{\mathrm{ab}} \rightarrow \pi^{S}\left(\mathcal{H} i l b_{X}^{n}, \widetilde{n x}\right)$. In $\S 5$, we use the criterion in [DM, Proposition $2.21]$ to show that this homomorphism is an isomorphism.

The hypothesis on the characteristic is needed in two places. First we use it in Proposition 3.3.3 to compute the power series ring at a certain closed point in $S^{n}(X)$; see equation (3.3.4). Here we need that $\operatorname{char}(k) \neq 2$. In Proposition 5.3.9 we show that the above homomorphism is a closed immersion. Here we need that $\operatorname{char}(k)>3$, which allows us to show that 
a certain sheaf on $S^{n}(X)$ is locally free on a very large open subset using Proposition 5.3.6.

We make a remark about the strategy of our proof. When $k=\mathbb{C}$, one easily checks that the map $\varphi_{*}$ induces an isomorphism of topological fundamental groups $\pi_{1}\left(\mathcal{H} i l b_{X}^{n}, \widetilde{n x}\right) \rightarrow \pi_{1}\left(S^{n}(X), n x\right)$, and then one shows that $\pi_{1}\left(S^{n}(X), n x\right)$ is isomorphic to $\pi_{1}(X, x)_{\mathrm{ab}}$, see [Bea83, Lemma 1, page 767]. In [BH15], the authors show that there are isomorphisms $\varphi_{*}: \pi^{\text {ét }}\left(\mathcal{H} i l b_{X}^{n}, \widetilde{n x}\right) \stackrel{\sim}{\rightarrow} \pi^{\text {ét }}\left(S^{n}(X), n x\right)$ and $\pi^{\text {ét }}\left(S^{n}(X), n x\right) \cong \pi^{\text {ét }}(X, x)_{\mathrm{ab}}$. In this article, however, we do not make the intermediate comparison with the group schemes corresponding to $S^{n}(X)$.

Note that $\mathcal{H} i l b_{X}^{1} \cong X$ and so we always assume that $n \geq 2$.

Acknowledgements. We thank Indranil Biswas for suggesting this question to us. We are very grateful to the referee for an extremely careful reading of this paper and for many useful suggestions.

\section{Fundamental Group Schemes}

In the rest of this article, unless mentioned otherwise, $k$ will denote an algebraically closed field of characteristic $p>3$.

2.1. Nori's fundamental group scheme. Let $X$ be a connected, proper and reduced $k$-scheme. We denote by $\mathrm{QCoh}(X)$ the category of quasicoherent sheaves of $\mathcal{O}_{X}$-modules on $X$. Consider the full subcategory $\operatorname{Vect}(X)$ of $\mathrm{QCoh}(X)$, whose objects are locally free coherent sheaves of $\mathcal{O}_{X}$-modules (vector bundles). A vector bundle $E$ is said to be finite if there are distinct non-zero polynomials $f, g \in \mathbb{Z}[t]$ with non-negative coefficients such that $f(E) \cong g(E)$.

Let $C$ be a connected smooth projective curve over $k$. The degree of a vector bundle $E$ on $C$ is defined to be the number

$$
\operatorname{deg}(E):=c_{1}(E) \cdot[C] .
$$

A vector bundle $E$ on $C$ is said to be semistable if for any non-zero proper subbundle $F \subset E$, we have

$$
\mu(F):=\frac{\operatorname{deg}(F)}{\operatorname{rank}(F)} \leq \frac{\operatorname{deg}(E)}{\operatorname{rank}(E)}=: \mu(E) .
$$

Definition 2.1.1. Let $X$ be a connected, projective and reduced $k$-scheme. Let $\mathcal{C}^{\mathrm{nf}}(X)$ denote the full subcategory of $\mathrm{QCoh}(X)$ whose objects are coherent sheaves $E$ on $X$ satisfying the following two conditions:

(1) E is locally free, and

(2) for any smooth projective curve $C$ over $k$ and any morphism $f$ : $C \longrightarrow X$, the vector bundle $f^{*} E$ is semistable of degree 0 . 
We call the objects of the category $\mathcal{C}^{\mathrm{nf}}(X)$ numerically flat vector bundles on $X$. In the literature these are also referred to as Nori semistable vector bundles and this category is also denoted by $N S(X)$; for example, see [EM11]. See also [Lan11, Remark 5.2]. However, we reserve the term semistable to refer to slope semistable.

Definition 2.1.2. A vector bundle $E$ on $X$ is said to be essentially finite if there exist two numerically flat vector bundles $V_{1}, V_{2}$ and finitely many finite vector bundles $F_{1}, \ldots, F_{n}$ on $X$ with $V_{2} \subseteq V_{1} \subseteq \bigoplus_{i=1}^{n} F_{i}$ such that $E \cong V_{1} / V_{2}$.

Unless otherwise specified, for any coherent sheaf $E$ on $X$, we denote by $E_{x}$ the fiber of $E$ at $x \in X$. Let $\operatorname{EF}(X)$ be the full subcategory of $\operatorname{Vect}(X)$ whose objects are essentially finite vector bundles on $X$. Let $\operatorname{Vect}_{k}$ be the category of finite dimensional $k$-vector spaces. Fix a closed point $x \in X$ and let

$$
T_{x}: \mathrm{EF}(X) \longrightarrow \operatorname{Vect}_{k}
$$

be the fiber functor defined by sending an object $E \in \mathrm{EF}(X)$ to its fiber $E_{x}$ at $x$. Then the quadruple $\left(\operatorname{EF}(X), \bigotimes, T_{x}, \mathcal{O}_{X}\right)$ is a neutral Tannakian category. The affine $k$-group scheme $\pi^{N}(X, x)$ representing the functor of $k$-algebras $\underline{\mathrm{Aut}}^{\otimes}\left(T_{x}\right)$ is called Nori's fundamental group scheme of $X$ based at $x$ (see [DM, Section 1] for definition of the functor $\underline{\mathrm{Aut}}^{\otimes}\left(T_{x}\right)$ ). It is shown in [Nor82, Proposition 4, p. 88] that $\pi^{N}(X, x) \cong \pi^{N}(X, y)$ for any two closed points $x, y \in X$.

2.2. $S$-fundamental group scheme. Let $E$ be a coherent sheaf on $X$. Denote by $E^{\vee}$ the sheaf $\mathscr{H} o m\left(E, \mathcal{O}_{X}\right)$. A coherent sheaf $E$ is said to be reflexive if the natural $\mathcal{O}_{X}$-module homomorphism $E \rightarrow E^{\vee \vee}$ is an isomorphism. Let $X$ be a connected smooth projective variety over $k$ of dimension $d$. Let $H$ be an ample divisor on $X$. The degree of a torsion free coherent sheaf $E$ on $X$ is defined to be the number

$$
\operatorname{deg}(E):=c_{1}(E) \cdot H^{d-1} .
$$

A sheaf $E$ on $X$ is said to be $H$-semistable if for any non-zero proper subsheaf $E^{\prime} \subset E$, we have

$$
\mu\left(E^{\prime}\right):=\frac{\operatorname{deg}\left(E^{\prime}\right)}{\operatorname{rank}\left(E^{\prime}\right)} \leq \frac{\operatorname{deg}(E)}{\operatorname{rank}(E)}=: \mu(E) .
$$

Let $F$ denote the absolute Frobenius morphism. We say that $E$ is strongly $H$-semistable its Frobenius pullbacks $\left(F^{n}\right)^{*} E$ are $H$-semistable, for all $n \geq 0$; see [Lan04, p. 252].

Definition 2.2.1. Let $X$ be a connected, smooth and projective variety over $k$ of dimension $d$ and let $H$ be an ample divisor on $X$. Let $\operatorname{Vect}_{0}^{s}(X)$ be the full subcategory of $\mathrm{QCoh}(X)$ whose objects are coherent sheaves $E$ on $X$ satisfying the following three conditions: 
(1) $E$ is reflexive,

(2) $E$ is strongly $H$-semistable, and

(3) $\operatorname{ch}_{1}(E) \cdot H^{d-1}=\operatorname{ch}_{2}(E) \cdot H^{d-2}=0$, where $\operatorname{ch}_{i}(E)$ denote the $i$-th Chern character of $E$, for all $i=1,2$.

Since $X$ is smooth, it follows from [Lan11, Proposition 4.1] that the objects of $\operatorname{Vect}_{0}^{s}(X)$ are in fact locally free sheaves and all of their Chern classes vanishes. Moreover, the category $\operatorname{Vect}_{0}^{s}(X)$ does not depend on the choice of ample divisor $H$ [Lan11, Proposition 4.5].

Assume that $X$ is smooth. Fix a $k$-valued point $x \in X$. Let $T_{x}$ : $\operatorname{Vect}_{0}^{s}(X) \longrightarrow \operatorname{Vect}_{k}$ be the fiber functor defined by sending an object $E$ of $\operatorname{Vect}_{0}^{S}(X)$ to its fiber $E_{x} \in \operatorname{Vect}_{k}$ at $x$. Then $\left(\operatorname{Vect}_{0}^{S}(X), \otimes, T_{x}, \mathcal{O}_{X}\right)$ is a neutral Tannaka category [Lan11, Proposition 5.5, p. 2096]. The affine $k$-group scheme $\pi^{S}(X, x)$ Tannaka dual to this category is called the $S$ fundamental group scheme of $X$ with base point $x$ [Lan11, Definition 6.1, p. 2097].

The following result may be well-known to experts, but we could not find a precise reference, so we include a proof. See also the proof of [Nor82, Chapter II, Proposition 4 (d), page 88].

Lemma 2.2.2. Let $X$ be a connected, smooth and projective $k$-scheme. Then $\pi^{S}\left(X, x_{1}\right) \cong \pi^{S}\left(X, x_{2}\right)$, for all $x_{1}, x_{2} \in X(k)$.

Proof. Since $\pi^{S}(X, x)$ is the affine $k$-group scheme representing the functor of $k$-algebras $\underline{\text { Aut }^{\otimes}}\left(T_{x}\right)$, where $T_{x}$ is the fiber functor $T_{x}: \operatorname{Vect}_{0}^{s}(X) \longrightarrow$ Vect $_{k}$, it suffices to show that, for any two points $x_{1}, x_{2} \in X(k)$, the fiber functors $T_{x_{1}}$ and $T_{x_{2}}$ are isomorphic. Given any object $\mathcal{V} \in \operatorname{Vect}_{0}^{s}(X)$, we need to define a natural $k$-linear isomorphism

$$
\eta_{\mathcal{V}}: T_{x_{1}}(\mathcal{V})=\mathcal{V}_{x_{1}} \longrightarrow \mathcal{V}_{x_{2}}=T_{x_{2}}(\mathcal{V})
$$

meaning that for any morphism $f: \mathcal{V} \rightarrow \mathcal{V}^{\prime}$ of objects in $\operatorname{Vect}_{0}^{s}(X)$, the following diagram should commute.

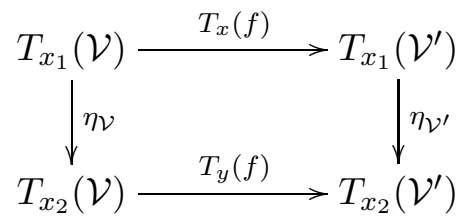

For any group scheme $H$ over $k$, denote by $\operatorname{Rep}_{k}(H)$ the category of representations of $H$ into finite dimensional $k$-vector spaces. Let $G=\pi^{S}\left(X, x_{1}\right)$. Then there is an equivalence of categories $\zeta: \operatorname{Vect}_{0}^{S}(X) \stackrel{\sim}{\longrightarrow} \operatorname{Rep}_{k}(G)$ and the inverse of this equivalence of categories defines a principal $G$-bundle $p: P \rightarrow X$, (see [Nor76, Proposition 2.9] for the construction), known as the $S$-universal cover of $X$ (see [Lan11, p. 2097]). This associates to a $G$-module $V$ an object $\mathcal{V}:=P \times^{G} V$ in the category $\operatorname{Vect}_{0}^{s}(X)$; moreover, 
any morphism $\mathcal{V} \rightarrow \mathcal{V}^{\prime}$ in the category $\operatorname{Vect}_{0}^{s}(X)$ comes from a $G$-module homomorphism $V \rightarrow V^{\prime}$ in $\operatorname{Rep}_{k}(G)$.

Fix two points $\tilde{x}_{1}, \tilde{x}_{2} \in P$ such that $p\left(\tilde{x}_{i}\right)=x_{i}$, for $i=1,2$. Then we have isomorphisms

$$
\xi_{i}: G \longrightarrow P_{x_{i}}, \quad i=1,2 .
$$

Let $\rho: G \rightarrow \mathrm{GL}(V)$ be a finite dimensional linear representation and let $\mathcal{V}:=P \times^{G} V$ be the associated vector bundle on $X$. Then we have $k$-linear isomorphisms

$$
\widetilde{\xi}_{i}: \mathcal{V}_{x_{i}}=P_{x_{i}} \times{ }^{G} V \stackrel{\xi_{i}^{-1}}{\cong} G \times{ }^{G} V \stackrel{\simeq}{\longrightarrow} V
$$

induced by $\xi_{i}$, for all $i=1,2$. This gives a $k$-linear isomorphism of the fibers

$$
\widetilde{\xi}_{2}^{-1} \circ \widetilde{\xi}_{1}=: \eta_{\mathcal{V}}: \mathcal{V}_{x_{1}} \longrightarrow \mathcal{V}_{x_{2}} \text {. }
$$

Since any homomorphism $f: \mathcal{V} \rightarrow \mathcal{V}^{\prime}$ of objects in $\operatorname{Vect}_{0}^{s}(X)$ comes from a $G$-module homomorphism $\tilde{f}: V \rightarrow V^{\prime}$, it follows from above construction that the above diagram in (2.2.3) commutes.

\section{Hilbert-Chow Morphism}

3.1. Hilbert scheme of length $n$ cycles. From now on we denote by $X$ an irreducible smooth projective surface over $k$. For an integer $n \geq 2$, let $S_{n}$ be the permutation group of $n$ symbols. Then $S_{n}$ acts on the product $X^{n}$ and the associated quotient $S^{n}(X)=X^{n} / S_{n}$ is a normal projective variety of dimension $2 n$ over $k$. Note that $S^{n}(X)$ is not smooth. Its smooth locus $S^{n}(X)_{\mathrm{sm}} \subset S^{n}(X)$ is the open dense subscheme consisting of reduced effective 0-cycles of length $n$ in $X$. Since $\operatorname{dim}_{k}(X)=2$, the singular locus $S^{n}(X)_{\text {sing }}:=S^{n}(X) \backslash S^{n}(X)_{\mathrm{sm}}$ is a closed subscheme of codimension 2 in $S^{n}(X)$.

Let $\mathcal{H} i l b_{X}^{n}$ be the Hilbert scheme parametrizing effective 0 -cycles of length $n$ in $X$. This is an irreducible smooth projective scheme of dimension $2 n$ over $k$. Consider the Hilbert-Chow morphism

$$
\varphi: \mathcal{H} i l b_{X}^{n} \longrightarrow S^{n}(X)
$$

given by sending $Z \in \mathcal{H} i l b_{X}^{n}$ to

$$
\sum_{p \in \operatorname{Supp}(Z)} \ell\left(\mathcal{O}_{Z, p}\right)[p] \in S^{n}(X),
$$

where

$$
\operatorname{Supp}(Z)=\left\{p \in X: \mathcal{O}_{Z, p} \neq 0\right\}
$$

denotes the support of the 0 -cycle $Z$ in $X$ and $\ell\left(\mathcal{O}_{Z, p}\right)$ the length of the local ring $\mathcal{O}_{Z, p}$ as a module over itself. It is well known that $\varphi$ is a proper morphism. 
3.2. Stratification of $S^{n}(X)$. A point $y \in S^{n}(X)$ can be written as

$$
\sum_{j=1}^{r} n_{j} x_{j},
$$

where $x_{1}, \ldots, x_{r} \in X$ are distinct points with multiplicities

$$
n_{1} \geq n_{2} \geq \cdots \geq n_{r} \in \mathbb{Z}_{>0},
$$

respectively, such that $\sum_{j=1}^{r} n_{j}=n$. The $r$-tuple of positive integers

$$
\left\langle n_{1}, n_{2}, \ldots, n_{r}\right\rangle
$$

is called the type of $y$. Let $Z_{\left\langle n_{1}, n_{2}, \ldots, n_{r}\right\rangle}$ denote the locus of points in $S^{n}(X)$ of type $\left\langle n_{1}, n_{2}, \ldots, n_{r}\right\rangle$. The fiber $\varphi^{-1}(y)$ has dimension $n-r$, for all $y \in$ $Z_{\left\langle n_{1}, n_{2}, \ldots, n_{r}\right\rangle}$ (see [Fog73, p. 667]). The dimension of the locus of points of type $\left\langle n_{1}, n_{2}, \ldots, n_{r}\right\rangle$ is $2 r$. From this the following lemma follows.

Lemma 3.2.2. The dimension of the subset $\varphi^{-1}\left(Z_{\left\langle n_{1}, n_{2}, \ldots, n_{r}\right\rangle}\right)$ is $n+r$.

3.3. Fibers of Hilbert-Chow morphism. Let $W \subset S^{n}(X)$ denote the open subset consisting of points of type $\langle 1,1,1, \ldots, 1\rangle$ and $\langle 2,1,1, \ldots, 1\rangle$. Let $V$ denote the open subset $\varphi^{-1}(W)$ and let

$$
\varphi: V \longrightarrow W
$$

be the restriction of the morphism $\varphi$ in (3.1.1) to $V$. It follows from Lemma 3.2.2 that the dimension of $\mathcal{H} i l b_{X}^{n} \backslash V$ is $n+n-2=2 n-2$ and hence $\operatorname{codim}_{\mathcal{H} i l b_{X}^{n}}\left(\mathcal{H} i l b_{X}^{n} \backslash V\right)=2$.

It was shown in [Fog73, Lemma 4.3, p. 668] that for any point $q \in S^{n}(X)$ of type $\langle 2,1,1, \ldots, 1\rangle$, the schematic fiber $\varphi^{-1}(q)$, with its reduced structure, is isomorphic to $\mathbb{P}_{k}^{1}$. We need that $\varphi^{-1}(q)$ is reduced. We could not find a precise reference for Proposition 3.3.3, which is well known to experts, so we include a proof.

First we recall the following result.

Lemma 3.3.2. Let I be an ideal of a commutative ring $A$ with identity. Let $A[I t]:=\bigoplus_{i=0}^{\infty} I^{i} t^{i} \subset A[t]$ be the Rees algebra of $I$ in the polynomial ring $A[t]$. Let $\pi: \operatorname{Proj}(A[I t]) \rightarrow \operatorname{Spec} A$ be the associated projective $A$-scheme. For an $A$-algebra $B$, consider the graded A-algebra structure on $A[I t] \otimes_{A} B$ given by $\left(A[I t] \otimes_{A} B\right)_{d}:=\left(I^{d} \otimes_{A} B\right) t^{d}$, for all $d \geq 0$. Then we have a canonical isomorphism of $A$-schemes

$$
\psi: \operatorname{Proj}\left(A[I t] \otimes_{A} B\right) \stackrel{\simeq}{\longrightarrow} \operatorname{Proj}(A[I t]) \times_{\operatorname{Spec} A} \operatorname{Spec} B .
$$

Proof. Follows from [Stk, Lemma 26.11.6., Tag 01MX].

Proposition 3.3.3. Assume that $\operatorname{char}(k) \neq 2$. Let $q \in W$ be a point of type $\langle 2,1,1, \ldots, 1\rangle$. The scheme theoretic fiber $\varphi^{-1}(q)$ is a reduced subscheme of $V$. 
Proof. Let $\tilde{q} \in X^{n}$ be a point such that $\tilde{q} \mapsto q$ under the natural map $\psi: X^{n} \rightarrow S^{n}(X)$. The formal neighbourhood of $\tilde{q}$ is given by the spectrum of the local ring

$$
\widehat{\mathcal{O}}_{X^{n}, \tilde{q}}=k\left[\left[x_{1}, y_{1}, x_{2}, y_{2}, \ldots, x_{n}, y_{n}\right]\right] .
$$

There is an inclusion $\widehat{\mathcal{O}}_{W, q} \hookrightarrow \widehat{\mathcal{O}}_{X^{n}}, \tilde{q}$. By the discussion in the paragraph just before $\left[\mathrm{FGI}^{+}\right.$05, Theorem 7.3.4, p. 170], we have

$$
\widehat{\mathcal{O}}_{W, q}=k\left[\left[u, v, w, x^{\prime}, y^{\prime}, x_{3}, y_{3}, \ldots, x_{n}, y_{n}\right]\right] /\left(u w-v^{2}\right),
$$

where $x=x_{1}-x_{2}, y=y_{1}-y_{2}, x^{\prime}=x_{1}+x_{2}, y^{\prime}=y_{1}+y_{2}, u=x^{2}, v=x y$ and $w=y^{2}$. Here we are using the assumption $\operatorname{char}(k) \neq 2$.

Let $Z \subset W$ denote the irreducible closed subset consisting of points of type $\langle 2,1,1, \ldots, 1\rangle$. Let $J$ denote the stalk at $q$ of the ideal sheaf of $Z$ in the local ring $\mathcal{O}_{W, q}$ and let $\widehat{J}$ denote its image in $\widehat{\mathcal{O}}_{W, q}$. Now $Z$ is contained in the image $\psi\left(X^{n-1}\right) \subset S^{n}(X)$, where the inclusion $X^{n-1} \hookrightarrow X^{n}$ is given by

$$
\left(x, x_{3}, x_{4}, \ldots, x_{n}\right) \longmapsto\left(x, x, x_{3}, \ldots, x_{n}\right) .
$$

Clearly, the ideal of $X^{n-1}$ in $\widehat{\mathcal{O}}_{X^{n}, \tilde{q}}$ is given by $x_{1}-x_{2}=y_{1}-y_{2}=0$. From this, we conclude that $\widehat{J}$ is the kernel of the composite homomorphism

$$
\widehat{\mathcal{O}}_{W, q} \hookrightarrow \widehat{\mathcal{O}}_{X^{n}, \tilde{q}} \rightarrow \widehat{\mathcal{O}}_{X^{n}, \tilde{q}} /(x, y),
$$

where $x=x_{1}-x_{2}$ and $y=y_{1}-y_{2}$. This proves that $\widehat{J}=(u, v, w)$.

By [Fog73, Lemma 4.4] the map $\varphi$ is the blowup of $W$ along $Z$. Let $\mathcal{O}_{W, q}[t J]$ denote the Rees algebra of the ideal $J$. By Lemma 3.3.2, the schematic fiber $\varphi^{-1}(q)$ is

$\operatorname{Proj}\left(\mathcal{O}_{W, q}[t J]\right) \times_{\operatorname{Spec}\left(\mathcal{O}_{W, q}\right)} \operatorname{Spec}\left(\mathcal{O}_{W, q} / \mathfrak{m}_{q}\right) \cong \operatorname{Proj}\left(\mathcal{O}_{W, q}[t J] \otimes_{\mathcal{O}_{W, q}}\left(\mathcal{O}_{W, q} / \mathfrak{m}_{q}\right)\right)$, where $\mathfrak{m}_{q}$ is the maximal ideal of the local ring $\mathcal{O}_{W, q}$ at $q$. It follows from the isomorphism

$$
\begin{aligned}
\mathcal{O}_{W, q}[t J] \otimes_{\mathcal{O}_{W, q}}\left(\mathcal{O}_{W, q} / \mathfrak{m}_{q}\right) & \cong \mathcal{O}_{W, q}[t J] \otimes_{\mathcal{O}_{W, q}}\left(\widehat{\mathcal{O}}_{W, q} / \widehat{\mathfrak{m}}_{q}\right) \\
& \cong \widehat{\mathcal{O}}_{W, q}\left[t \widehat{J]} \otimes_{\widehat{\mathcal{O}}_{W, q}}\left(\widehat{\mathcal{O}}_{W, q} / \widehat{\mathfrak{m}}_{q}\right)\right.
\end{aligned}
$$

that the schematic fiber $\varphi^{-1}(q)$ is

$\operatorname{Proj}\left(\widehat{\mathcal{O}}_{W, q}[t \widehat{J}] \otimes_{\widehat{\mathcal{O}}_{W, q}}\left(\widehat{\mathcal{O}}_{W, q} / \widehat{\mathfrak{m}}_{q}\right)\right) \cong \operatorname{Proj}\left(\widehat{\mathcal{O}}_{W, q}[t \widehat{J}]\right) \times_{\operatorname{Spec}\left(\widehat{\mathcal{O}}_{W, q}\right)} \operatorname{Spec}\left(\widehat{\mathcal{O}}_{W, q} / \widehat{\mathfrak{m}}_{q}\right)$.

Write

$$
A:=\widehat{\mathcal{O}}_{W, q}=k\left[\left[u, v, w, x^{\prime}, y^{\prime}, x_{3}, y_{3}, \ldots, x_{n}, y_{n}\right]\right] /\left(u w-v^{2}\right) .
$$

It is clear that the maximal ideal $\mathfrak{m}:=\widehat{\mathfrak{m}}_{q}$ of $A$ is given by

$$
\mathfrak{m}=\left(u, v, w, x^{\prime}, y^{\prime}, x_{3}, y_{3}, \ldots, x_{n}, y_{n}\right) A .
$$


First let us understand the scheme $\operatorname{Proj}(A[t \widehat{J}])$. This scheme is covered by affine open subsets given by Spec of the following three affine $k$-algebras:

$$
\begin{aligned}
& R_{1}:=\left(k\left[\left[u, v, w, x^{\prime}, y^{\prime}, x_{3}, y_{3}, \ldots, x_{n}, y_{n}\right]\right] /\left(u w-v^{2}\right)\right)\left[\frac{v}{u}, \frac{w}{u}\right], \\
& R_{2}:=\left(k\left[\left[u, v, w, x^{\prime}, y^{\prime}, x_{3}, y_{3}, \ldots, x_{n}, y_{n}\right]\right] /\left(u w-v^{2}\right)\right)\left[\frac{u}{v}, \frac{w}{v}\right], \\
& R_{3}:=\left(k\left[\left[u, v, w, x^{\prime}, y^{\prime}, x_{3}, y_{3}, \ldots, x_{n}, y_{n}\right]\right] /\left(u w-v^{2}\right)\right)\left[\frac{u}{w}, \frac{v}{w}\right] .
\end{aligned}
$$

Let us first consider the ring $R_{1}$. In this ring, $\frac{w}{u}=\frac{w u}{u^{2}}=\frac{v^{2}}{u^{2}}$. Therefore, we get that

$$
R_{1}=\left(k\left[\left[u, v, w, x^{\prime}, y^{\prime}, x_{3}, y_{3}, \ldots, x_{n}, y_{n}\right]\right] /\left(u w-v^{2}\right)\right)\left[\frac{v}{u}\right] .
$$

Similarly, since $\frac{u}{w}=\frac{u w}{w^{2}}=\frac{v^{2}}{w^{2}}$, we get that

$$
R_{3}=\left(k\left[\left[u, v, w, x^{\prime}, y^{\prime}, x_{3}, y_{3}, \ldots, x_{n}, y_{n}\right]\right] /\left(u w-v^{2}\right)\right)\left[\frac{v}{w}\right] .
$$

Further, in $R_{2}$ we have $\frac{u}{v} \frac{w}{v}=1$. Therefore,

$$
R_{2}=\left(k\left[\left[u, v, w, x^{\prime}, y^{\prime}, x_{3}, y_{3}, \ldots, x_{n}, y_{n}\right]\right] /\left(u w-v^{2}\right)\right)\left[\frac{v}{u}, \frac{u}{v}\right] .
$$

It is now clear that the scheme $\operatorname{Proj}(A[t \widehat{J}])$ is covered by $\operatorname{Spec} R_{1}$ and Spec $R_{3}$, since $\operatorname{Spec} R_{2}$ is an open subset of each of these. Now we need to compute

$$
R_{1} \otimes_{A}(A / \mathfrak{m}) \quad \text { and } \quad R_{3} \otimes_{A}(A / \mathfrak{m}) .
$$

Let us first write

$$
R_{1}=A[T] /(T u-v) .
$$

Now note that $(T u-v) \subset \mathfrak{m} A[T]$ since $\mathfrak{m}$ contains $u$ and $v$. Therefore we get

$$
\begin{aligned}
R_{1} \otimes_{A}(A / \mathfrak{m}) & \cong R_{1} / \mathfrak{m} R_{1} \\
& \cong A[T] / \mathfrak{m} A[T] \cong k[T] .
\end{aligned}
$$

Similarly, we have $R_{3} \otimes_{A}(A / \mathfrak{m}) \cong k[T]$. Thus we have proved that the scheme theoretic fiber $\varphi^{-1}(q)$ is reduced and is isomorphic to $\mathbb{P}_{k}^{1}$.

\section{Homomorphism of $S$-Fundamental Group Schemes}

Fix a closed point $x \in X$ and let $\widetilde{n x}$ be a point in $\mathcal{H} i l b_{X}^{n}$ mapping to $n x \in$ $S^{n}(X)$. In this section we construct a homomorphism of $S$-fundamental group schemes

$$
\pi^{S}(X, x)_{\mathrm{ab}} \longrightarrow \pi^{S}\left(\mathcal{H} i b_{X}^{n}, \widetilde{n x}\right),
$$

where $\pi^{S}(X, x)_{\mathrm{ab}}$ is the abelianization of $\pi^{S}(X, x)$. 
4.1. A group scheme theoretic lemma. We need the following group scheme theoretic result for later use. First recall the definition of the derived subgroup $\mathscr{D} G$ as given in [Wat79, $\S 10.1$ ]. It is a closed normal subgroup. It follows from the main Theorem in [Wat79, §16.3] that there is a quotient $\alpha: G \rightarrow G_{\text {ab }}$ whose kernel is precisely $\mathscr{D} G$. It is clear that $G_{\mathrm{ab}}$ is an abelian affine group scheme.

Lemma 4.1.1. Let $G$ and $H$ be two group schemes over $k$. For an integer $n \geq 2$, we denote by $G^{n}$ the group scheme $G \times \cdots \times G$ (= the $n$-fold product of $G$ with itself). Then $S_{n}$ acts on $G^{n}$ by permuting the factors. Let $f_{0}$ be the following composite group homomorphism

$$
f_{0}: G^{n} \stackrel{\alpha^{n}}{\longrightarrow}\left(G_{\mathrm{ab}}\right)^{n} \stackrel{m}{\longrightarrow} G_{\mathrm{ab}},
$$

where $m$ denotes the multiplication homomorphism. Then a homomorphism of k-group schemes $f: G^{n} \longrightarrow H$ is $S_{n}$-invariant if and only if there is a homomorphism $\tilde{f}: G_{\mathrm{ab}} \longrightarrow H$ of affine $k$-group schemes such that $\tilde{f} \circ f_{0}=f$. In other words, the following diagram commutes.

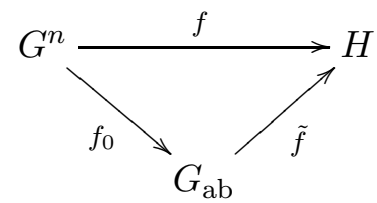

Proof. For any $k$-group scheme $G$, we denote by

- $m_{G}: G \times G \longrightarrow G$ the multiplication morphism of $G$,

- $i_{G}: G \longrightarrow G$ the inversion morphism of $G$, and

- $e_{G} \in G(k)$ the identity of $G$.

We sketch the proof for $n=2$; the general case is similar and left to the reader as an exercise. We have a homomorphism $f: G \times G \longrightarrow H$ such that $f \circ \sigma=f$, where $\sigma: G \times G \rightarrow G \times G$ is the homomorphism switching the factors. Let $p_{1}, p_{2}: G \times G \rightarrow G$ denote the projections onto the first and second factors, respectively. Then one can easily check that

$$
\begin{aligned}
f \circ\left(m_{G}, e_{G}\right) & =f \circ m_{G \times G} \circ\left(\left(p_{1}, e\right),\left(p_{2}, e\right)\right) \\
& =m_{H} \circ\left(f \circ\left(p_{1}, e\right), f \circ\left(p_{2}, e\right)\right) \\
& =m_{H} \circ\left(f \circ\left(p_{1}, e\right), f \circ\left(e, p_{2}\right)\right) \\
& =f \circ\left(p_{1}, p_{2}\right) \\
& =f \circ\left(m_{G} \circ \sigma, e_{G}\right) .
\end{aligned}
$$

Using this it easily follows that

$$
f \circ m_{G \times G}\left(\left(m_{G}, e_{G}\right),\left(i_{G} \circ m_{G} \circ \sigma, e_{G}\right)\right)=e_{H} .
$$

Now one easily concludes that $f$ factors through the map $G \times G \rightarrow G_{\mathrm{ab}} \times G_{\mathrm{ab}}$. Let $\Delta^{\prime}: G \rightarrow G \times G$ denote the map $g \mapsto\left(g, g^{-1}\right)$. Then one checks easily that $f \circ \Delta^{\prime}=e_{H}$. From these the lemma follows. 
A vector bundle $E$ on $X^{n}$ is said to be $S_{n}$-invariant if $\sigma^{*} E \cong E$, for all $\sigma \in S_{n} \subseteq \operatorname{Aut}\left(X^{n}\right)$.

Corollary 4.1.3. Any vector bundle in the category $\operatorname{Vect}_{0}^{s}\left(X^{n}\right)$, associated to a representation of $\pi^{S}(X, x)^{n}$ which factors through $\pi^{S}(X, x)_{\mathrm{ab}}$ (see the statement of Lemma 4.1.1), is $S_{n}$-invariant.

4.2. A functor between Tannakian categories. Given a numerically flat vector bundle $E$ on $\mathcal{H} i l b_{X}^{n}$, we want to associate to it a numerically flat vector bundle $\mathcal{G}$ on $X^{n}$. We first associate to $E$ a reflexive sheaf $\mathcal{G}$ on $X^{n}$ and then use the criterion in [Lan12, Theorem 2.2] to show that $\mathcal{G}$ is numerically flat. We recall the criterion here for the benefit of the reader.

Theorem. [Lan12, Theorem 2.2] Let $X$ be a smooth projective $k$-variety of dimension $d$. Let $H$ be an ample divisor on $X$ and let $E$ be a coherent sheaf on $X$. Then $E$ is numerically flat if and only if $E$ is a strongly $H$-semistable reflexive sheaf with $\mathrm{ch}_{1}(E) \cdot H^{d-1}=\operatorname{ch}_{2}(E) \cdot H^{d-2}=0$.

Recall that $W \subset S^{n}(X)$ is the open subset consisting of points of type $\langle 1,1,1, \ldots, 1\rangle$ and $\langle 2,1,1, \ldots, 1\rangle$, and $V$ is the open subset $\varphi^{-1}(W) \subset \mathcal{H} i l b_{X}^{n}$, where $\varphi$ is the Hilbert-Chow morphism.

Proposition 4.2.1. Let $E$ be a numerically flat vector bundle of rank $r$ on $\mathcal{H} i l b_{X}^{n}$. Then $\varphi_{*}\left(\left.E\right|_{V}\right)$ is a locally free coherent sheaf on $W$. Moreover, the natural map

$$
\left.\varphi^{*} \varphi_{*}\left(\left.E\right|_{V}\right) \longrightarrow E\right|_{V}
$$

is an isomorphism.

Proof. Let $q \in W$ be a point of type $\langle 2,1,1, \ldots, 1\rangle$. Let $\mathcal{I} \subset \mathcal{O}_{V}$ denote the reduced sheaf of ideals of the closed subscheme $\varphi^{-1}(q)$. Let $\mathscr{I}_{q}$ be the ideal sheaf of the closed point $q \in W$. For each integer $n \geq 1$, let $\mathscr{I}_{q}^{n}$ be the ideal sheaf of the $n$-th order thickening of $q$ in $W$. By Proposition 3.3.3 we have

$$
\mathcal{I}=\mathscr{I}_{q} \mathcal{O}_{V}
$$

For each integer $n \geq 1$, let $Y_{n}$ denote the closed subscheme of $V$ corresponding to the sheaf of ideals $\mathcal{I}^{n}$. Since $E$ is numerically flat and $Y_{1} \cong \mathbb{P}_{k}^{1}$ (see Proposition 3.3.3), it follows that the restriction of $E$ to $Y_{1}$ is trivial.

Consider the following short exact sequence of sheaves on $V$

$$
\left.0 \longrightarrow \mathcal{I} \otimes E \longrightarrow E \longrightarrow E\right|_{Y_{1}} \longrightarrow 0
$$

Applying $\varphi_{*}$ to it we get the following exact sequence of sheaves on $W$.

$$
\varphi_{*}(E) \longrightarrow H^{0}\left(Y_{1},\left.E\right|_{Y_{1}}\right) \longrightarrow R^{1} \varphi_{*}(\mathcal{I} \otimes E) .
$$

We claim that the completion of $R^{1} \varphi_{*}(\mathcal{I} \otimes E)$ at the maximal ideal $\mathfrak{m}_{q}$ of $q$ is 0. By the Theorem on Formal Functions (see [Har77, Chapter III, Theorem 11.1]), we have

$$
\left(R^{1} \varphi_{*}(\mathcal{I} \otimes E)\right)^{\wedge} \cong \lim _{\longleftarrow} H^{1}\left(Y_{n}, \mathcal{I} \otimes E \otimes \mathcal{O}_{V} / \mathcal{I}^{n}\right) .
$$


We will prove by induction on $n$ that $H^{1}\left(Y_{n}, \mathcal{I} \otimes E \otimes \mathcal{O}_{V} / \mathcal{I}^{n}\right)=0$. Since $\mathcal{I}=\mathscr{I}_{q} \mathcal{O}_{V}$, it follows that there is a surjection

$$
\left(\mathfrak{m}_{q}^{n} / \mathfrak{m}_{q}^{n+1}\right) \otimes_{\mathcal{O}_{W, q}} \mathcal{O}_{V} \cong \mathscr{I}_{q}^{n} / \mathscr{I}_{q}^{n+1} \otimes_{\mathcal{O}_{W}} \mathcal{O}_{V} \rightarrow \mathcal{I}^{n} / \mathcal{I}^{n+1},
$$

where $\mathcal{O}_{W, q}$ is the stalk of $\mathcal{O}_{W}$ at $q$. The locally free sheaf $\mathcal{I}^{n} / \mathcal{I}^{n+1}$ on $Y_{1} \cong \mathbb{P}^{1}$ is a direct sum of line bundles. It follows that each of these line bundle has degree $\geq 0$. For $n=1$, the base case of induction, we have

$$
H^{1}\left(Y_{1}, \mathcal{I} \otimes E \otimes \mathcal{O}_{V} / \mathcal{I}\right)=H^{1}\left(Y_{1}, \mathcal{I} / \mathcal{I}^{2} \otimes E_{1}\right)=0 .
$$

Assume that we have proved the assertion for $n$. Then the assertion for $n+1$ follows from the long exact cohomology sequence attached to the short exact sequence of sheaves on $Y_{n+1}$

$$
0 \longrightarrow\left(\mathcal{I}^{n+1} / \mathcal{I}^{n+2}\right) \otimes E \longrightarrow\left(\mathcal{I} / \mathcal{I}^{n+2}\right) \otimes E \longrightarrow\left(\mathcal{I} / \mathcal{I}^{n+1}\right) \otimes E \longrightarrow 0 .
$$

This proves the claim that $R^{1} \varphi_{*}(\mathcal{I} \otimes E)$ at the maximal ideal $\mathfrak{m}_{q}$ of $q$ is 0 .

This proves that the natural map

$$
\varphi_{*}(E) \longrightarrow H^{0}\left(Y_{1},\left.E\right|_{Y_{1}}\right)
$$

in (4.2.4) is surjective in a neighborhood around $q$. Let $s_{1}, s_{2} \ldots, s_{r}$ be a basis for $H^{0}\left(Y_{1},\left.E\right|_{Y_{1}}\right)$. Let $\operatorname{Spec}(A)$ be an affine neighborhood of $q$ on which the map in (4.2.6) is surjective. Choosing lifts $\tilde{s}_{i} \in \Gamma\left(\operatorname{Spec}(A), \varphi_{*}(E)\right)$ of $s_{i}$, we get a homomorphism

$$
\mathcal{O}_{V}^{\oplus r} \longrightarrow E
$$

over $\varphi^{-1}(\operatorname{Spec}(A))$, which is a surjection over the fiber $Y_{1}$. Since $\varphi$ is proper, it follows that there is a smaller affine neighborhood $W_{0}$ of $q$ over which there is an isomorphism $\mathcal{O}_{V_{0}}^{\oplus r} \stackrel{\sim}{\rightarrow} E$, where $V_{0}=\varphi^{-1}\left(W_{0}\right)$. Applying $\varphi_{*}$, using normality of $S^{n}(X)$ and that $\varphi$ is birational, the Proposition follows.

Corollary 4.2.8. Let $F$ denote the absolute Frobenius morphism. With the above notations, we have an isomorphism $F^{*} \varphi_{*}\left(\left.E\right|_{V}\right) \stackrel{\sim}{\longrightarrow} \varphi_{*}\left(\left.F^{*} E\right|_{V}\right)$.

Proof. Since $F^{*} E$ is numerically flat, it follows that both these sheaves are locally free of the same rank. It suffices to show that the natural map

$$
F^{*} \varphi_{*}\left(\left.E\right|_{V}\right) \longrightarrow \varphi_{*}\left(\left.F^{*} E\right|_{V}\right)
$$

is surjective. This is clear over the smooth locus of $S^{n}(X)$ since $F$ is faithfully flat over the smooth locus. Let $q \in W$ be a point of type $\langle 2,1,1 \ldots, 1\rangle$. It follows from Proposition 3.3.3 that the restriction of $F^{*} \varphi_{*}\left(\left.E\right|_{V}\right)$ to $q$ is naturally isomorphic to $H^{0}\left(Y_{1}, E_{1}\right)$ and the restriction of $\varphi_{*}\left(\left.F^{*} E\right|_{V}\right)$ at $q$ is naturally isomorphic to $H^{0}\left(Y_{1}, F^{*} E_{1}\right)$. The restriction to $q$ of the natural homomorphism in (4.2.9) is the map

$$
F^{*}: H^{0}\left(Y_{1}, E_{1}\right) \longrightarrow H^{0}\left(Y_{1}, F^{*} E_{1}\right)
$$

which is a surjection. From this the Corollary follows. 
Recall the quotient map $\psi: X^{n} \longrightarrow S^{n}(X)$ defined in (3.1.1). Let $j$ : $\psi^{-1}(W) \hookrightarrow X^{n}$ denote the inclusion. Recall that the category $\mathcal{C}^{\text {nf }}(X)$ is defined in Definition 2.1.1.

Proposition 4.2.10. If $E$ is an object of $\mathcal{C}^{\operatorname{nf}}\left(\mathcal{H} i l b_{X}^{n}\right)$, then

$$
\mathscr{G}(E):=\left(j_{*} \psi^{*} \varphi_{*}\left(\left.E\right|_{V}\right)\right)^{\vee \vee}
$$

is an object of $\mathcal{C}^{\operatorname{nf}}\left(X^{n}\right)$.

Proof. It is proved in Proposition 4.2.1 that $\varphi_{*}(E)$ is locally free on $W$. Since $X^{n} \backslash \psi^{-1}(W)$ has codimension $\geq 4$, it follows that

$$
\mathscr{G}(E):=\left(j_{*} \psi^{*} \varphi_{*}\left(\left.E\right|_{V}\right)\right)^{\vee \vee}
$$

is a coherent reflexive sheaf on $X^{n}$. For notational simplicity, we denote by $\mathcal{G}$ the sheaf $\mathscr{G}(E)$. Note that $\left.\mathcal{G}\right|_{\psi^{-1}(W)}=\psi^{*} \varphi_{*}\left(\left.E\right|_{V}\right)$ is locally free.

Choose $m \gg 0$ so that $m H$ is very ample. Choose general hyperplanes $H_{1}, \ldots, H_{d-1} \in|m H|$ so that $C=H_{1} \cap H_{2} \cap \cdots \cap H_{d-1} \stackrel{i}{\hookrightarrow} \psi^{-1}(W)$ is a smooth complete intersection curve whose image $\psi(C)$ lies in the smooth locus of $S^{n}(X)$. Since $\varphi: \varphi^{-1}\left(S^{n}(X)_{\mathrm{sm}}\right) \rightarrow S^{n}(X)_{\mathrm{sm}}$ is an isomorphism, we can lift $i$ to a morphism $\tilde{i}$ which makes the following diagram commute.

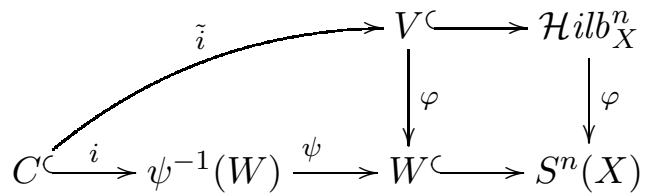

It follows from Proposition 4.2.1 that

$$
i^{*} \mathcal{G} \cong \tilde{i}^{*} \varphi^{*} \varphi_{*}\left(\left.E\right|_{V}\right) \cong \tilde{i}^{*}\left(\left.E\right|_{V}\right) .
$$

Since $E$ is in $\mathcal{C}^{\mathrm{nf}}\left(\mathcal{H} i b_{X}^{n}\right)$ it follows that $i^{*} \mathcal{G}$ is semistable of degree 0 . This shows that $\mathcal{G}$ is $H$-semistable.

In Corollary 4.2 .8 we proved that the locally free sheaves $F^{*} \varphi_{*}\left(\left.E\right|_{V}\right)$ and $\varphi_{*}\left(\left.F^{*} E\right|_{V}\right)$ are isomorphic. Since $X^{n}$ is smooth the Frobenius is faithfully flat and so $F^{*} \mathcal{G}$ is reflexive (use the characterization that a coherent module $M$ over a local ring $A$ is reflexive iff it sits in a short exact sequence $0 \rightarrow$ $\left.M \rightarrow A^{\oplus r} \rightarrow A^{\oplus s}\right)$. The restriction of $F^{*} \mathcal{G}$ on $\psi^{-1}(W)$ is

$$
F^{*} \psi^{*} \varphi_{*}\left(\left.E\right|_{V}\right) \cong \psi^{*} F^{*} \varphi_{*}\left(\left.E\right|_{V}\right) \cong \psi^{*} \varphi_{*}\left(\left.F^{*} E\right|_{V}\right) .
$$

Since the reflexive extension on $X^{n}$ is unique (see [Har80, Proposition 1.6, p. 126]), we conclude that

$$
F^{*} \mathcal{G} \cong\left(j_{*}\left(\psi^{*} \varphi_{*}\left(\left.F^{*} E\right|_{V}\right)\right)\right)^{\vee \vee} .
$$

Since $E \in \mathcal{C}^{\operatorname{nf}}\left(\mathcal{H} i l b_{X}^{n}\right)$ we have $F^{*} E \in \mathcal{C}^{\text {nf }}\left(\mathcal{H} i l b_{X}^{n}\right)$; then following the arguments in the preceding paragraph, we see that $F^{*} \mathcal{G}$ is $H$-semistable. In this way we can show that all Frobenius pullbacks of $\mathcal{G}$ are semistable. This shows that $\mathcal{G}$ is strongly $H$-semistable. 
It is clear from above that $\operatorname{ch}_{1}(\mathcal{G}) \cdot H^{d-1}=0$. Choose general hyperplanes $H_{1}, \ldots, H_{d-2}$ in the linear system $|m H|$ so that

$$
S=H_{1} \cap H_{2} \cap \cdots \cap H_{d-2} \subset \psi^{-1}(W)
$$

is a smooth surface. We can do this since $X^{n} \backslash \psi^{-1}(W)$ has codimension $\geq 4$. It suffices to show that $\operatorname{ch}_{2}\left(\left.\mathcal{G}\right|_{S}\right)=0$. Now $\left.\mathcal{G}\right|_{S}$ is locally free as $S \subset \psi^{-1}(W)$ and $\mathcal{G}$ is locally free on $\psi^{-1}(W)$. Therefore, in view of [Lan12, Theorem 2.2], it suffices to show that $\left.\mathcal{G}\right|_{S} \in \mathcal{C}^{\text {nf }}(S)$. But this follows from the arguments as in the second paragraph of this proof. Therefore, we have $\mathcal{G} \in \operatorname{Vect}_{0}^{s}\left(X^{n}\right)$ and hence by [Lan12, Theorem 2.2] $\mathcal{G}$ is locally free and is in $\mathcal{C}^{\operatorname{nf}}\left(X^{n}\right)$. This proves the proposition.

Proposition 4.2.12. With the above notations,

$$
\mathscr{G}: \mathcal{C}^{\mathrm{nf}}\left(\mathcal{H} i l b_{X}^{n}\right) \longrightarrow \mathcal{C}^{\mathrm{nf}}\left(X^{n}\right)
$$

is a additive tensor functor.

Proof. First we show that $\mathscr{G}$ is a functor. Let $f: E \rightarrow E^{\prime}$ be a morphism in the category $\mathcal{C}^{\mathrm{nf}}\left(\mathcal{H} i l b_{X}^{n}\right)$. We need to find a canonical morphism $\mathscr{G}(f)$ : $\mathscr{G}(E) \rightarrow \mathscr{G}\left(E^{\prime}\right)$ in $\mathcal{C}^{\mathrm{nf}}\left(X^{n}\right)$. There is a morphism $\psi^{*} \varphi_{*}(f):\left.\mathscr{G}(E)\right|_{\psi^{-1}(W)} \rightarrow$ $\left.\mathscr{G}\left(E^{\prime}\right)\right|_{\psi^{-1}(W)}$. Since $X^{n} \backslash \psi^{-1}(W)$ has codimension $\geq 4$ and $\mathscr{G}(E), \mathscr{G}\left(E^{\prime}\right)$ are locally free, it follows that this morphism extends uniquely to give a morphism $\mathscr{G}(E) \rightarrow \mathscr{G}\left(E^{\prime}\right)$.

The bundles $\mathscr{G}\left(E \oplus E^{\prime}\right)$ and $\mathscr{G}(E) \oplus \mathscr{G}\left(E^{\prime}\right)$ are naturally isomorphic on $\psi^{-1}(W)$ and so they are naturally isomorphic. Similarly, $\mathscr{G}\left(E \otimes E^{\prime}\right)$ is naturally isomorphic to $\mathscr{G}(E) \otimes \mathscr{G}\left(E^{\prime}\right)$.

4.3. Homomorphism of group schemes. Fix distinct $k$-valued points $x_{1}, \ldots, x_{n} \in X(k)$ of $X$. Let $\tilde{x} \in \mathcal{H} i l b_{X}^{n}(k)$ be such that $\varphi(\widetilde{x})=\psi\left(x_{1}, \cdots, x_{n}\right) \in$ $S^{n}(X)_{\mathrm{sm}}$. For any locally free sheaf $E$ on $\mathcal{H} i l b_{X}^{n}$, there are natural isomorphisms of fibers

$$
E_{\tilde{x}} \cong\left(\varphi_{*} E\right)_{\varphi(\tilde{x})} \cong\left(\psi^{*} \varphi_{*}(E)\right)_{\left(x_{1}, x_{2}, \ldots, x_{n}\right)} .
$$

Consider the following diagram.

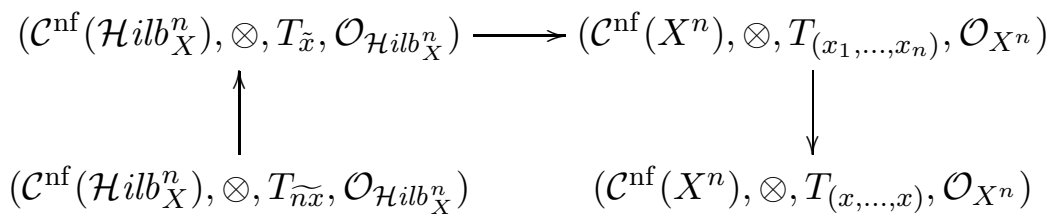

The horizontal arrow is a morphism of Tannakian categories due to Propositions 4.2.10 and 4.2.12. The two vertical arrows are due to Lemma 2.2.2. Thus, we get a homomorphism of $S$-fundamental group schemes

$$
f: \pi^{S}\left(X^{n},(x, \ldots, x)\right) \longrightarrow \pi^{S}\left(\mathcal{H} i l b_{X}^{n}, \widetilde{n x}\right) .
$$


For $\sigma \in S_{n}$ we get an automorphism $\sigma_{*}$ of $\pi^{S}\left(X^{n},(x, \ldots, x)\right)$. It is easily checked that $f \circ \sigma_{*}=f$. By [Lan12, Theorem 4.1, p. 842] there is an isomorphism

$$
\pi^{S}\left(X^{n},(x, \ldots, x)\right) \stackrel{\sim}{\longrightarrow} \pi^{S}(X, x) \times_{k} \cdots \times_{k} \pi^{S}(X, x) .
$$

By abuse of notation, denote the composite of $f$ and the inverse of this isomorphism by $f$. Thus, we have a homomorphism

$$
f: \pi^{S}(X, x) \times_{k} \cdots \times_{k} \pi^{S}(X, x) \longrightarrow \pi^{S}\left(\mathcal{H} i l b_{X}^{n}, \widetilde{n x}\right)
$$

which satisfies $f \circ \sigma_{*}=f$. It follows from Lemma 4.1.1 that the homomorphism of the $S$-fundamental group schemes in (4.3.1) factors through a homomorphism

$$
\tilde{f}: \pi^{S}(X, x)_{\mathrm{ab}} \longrightarrow \pi^{S}\left(\mathcal{H} i l b_{X}^{n}, \widetilde{n x}\right) .
$$

This completes the construction of our homomorphism of $k$-group schemes.

\section{Isomorphism of Group Schemes}

In this section we use [DM, Proposition 2.21] to show that the homomorphism $\tilde{f}$ in (4.3.2) is an isomorphism.

5.1. $S_{n}$-invariant line bundles. We begin with a discussion on why a numerically flat $S_{n}$-invariant line bundle on $X^{n}$ descends to a line bundle on $S^{n}(X)$. A more general result is proved in [Fog77, Proposition 3.6]. For the benefit of the authors and the reader we include a proof of the statement that we need.

Proposition 5.1.1. Let $\mathcal{L}$ be a numerically flat $S_{n}$-invariant line bundle on $X^{n}$. Then there is a numerically flat line bundle $L^{\prime}$ on $S^{n}(X)$ such that $\psi^{*} L^{\prime}=\mathcal{L}$.

Proof. The assertion that $L^{\prime}$, if it exists, is numerically flat follows easily. We now prove its existence.

Let $\mathrm{Pic}^{\tau}$ denote the subscheme of the Picard scheme whose closed points parametrize numerically trivial line bundles. By [Lan12, Corollary 4.7] we have

$$
\left(\operatorname{Pic}^{\tau}\left(X^{n}\right)\right)_{\mathrm{red}}=\prod_{i=1}^{n}\left(\operatorname{Pic}^{\tau}(X)\right)_{\mathrm{red}}
$$

Thus, there is a numerically trivial line bundle $L_{0}$ on $X$ such that $\mathcal{L}=$ $\bigotimes_{i=1}^{n} p_{i}^{*} L_{0}$.

The rest of the proof is a more detailed version of the first part of the proof in [Fog77, Proposition 3.6]. Let $H \subset S_{n}$ denote the subgroup Stab(1). Let $D \subset X$ be an ample divisor such that $L_{0}$ is trivial on $U=X \backslash D$. Let $s \in \Gamma\left(U, L_{0}\right)$ be a global section which generates $L_{0}$ over $U$. Then $p_{1}^{*} s$ is a generating section of $p_{1}^{*} L_{0}$ over the open subset $U \times X \times \ldots \times X$ and this 
section is invariant under the action of the subgroup $H$. In particular, the section $p_{1}^{*} s$ also generates the line bundle $p_{1}^{*} L_{0}$ over the smaller open subset $U^{n}$ and is invariant under the action of the subgroup $H$.

Given $\left(x_{1}, x_{2}, \ldots, x_{n}\right) \in X^{n}$, let $D$ be an ample divisor in $X$ which does not contain any of the $x_{i}$. If $U=X \backslash D$, then it is clear that $\left(x_{1}, \ldots, x_{n}\right)$ is in the $S_{n}$-invariant open subset $U^{n}$. Thus, we can cover $X^{n}$ by open subsets of this type. Using this observation, we can find a finite collection of ample divisors $D_{\alpha} \subset X$ (set $\left.U_{\alpha}=X \backslash D_{\alpha}\right)$ and sections $s_{\alpha} \in \Gamma\left(U_{\alpha}, L_{0}\right)$ such that

(1) $t_{\alpha}:=p_{1}^{*} s_{\alpha}$ generates $p_{1}^{*} L_{0}$ on the open subset $U_{\alpha}^{n}$,

(2) $t_{\alpha}$ is invariant under $H$, and

(3) $X^{n}=\bigcup_{\alpha} U_{\alpha}^{n}$.

Define functions $f_{\alpha \beta} \in \mathcal{O}_{X}\left(U_{\alpha}^{n} \cap U_{\beta}^{n}\right)^{\times}$by

$$
t_{\alpha}=f_{\alpha \beta} t_{\beta} .
$$

It follows that $f_{\alpha \beta}$ are invariant under $H$. Let $\sigma_{i}:=(1, i)$ for $1 \leq i \leq n$ be left coset representatives of $H$ in $G$. The functions $\prod_{i=1}^{n} \sigma_{i}^{*}\left(f_{\alpha \beta}\right)$ are clearly invariant under $S_{n}$ and satisfy the cocyle condition. Let $V_{\alpha} \subset S^{n}(X)$ be $\psi\left(U_{\alpha}^{n}\right)$. It is clear that $V_{\alpha}$ is open and $\psi^{-1}\left(V_{\alpha}\right)=U_{\alpha}^{n}$. Thus, using the above cocycle we get a line bundle on $S^{n}(X)$ which is trivial on $V_{\alpha}$. It is clear that the pullback of this line bundle is isomorphic to $\bigotimes_{i=1}^{n} p_{i}^{*} L_{0}$, which completes the proof of the proposition.

5.2. Faithfully flatness. In this subsection we use to show that the homomorphism $\tilde{f}$ in (4.3.2) is faithfully flat. We begin by recalling [DM, Proposition 2.21] for the convenience of the reader.

Let $\theta: G \longrightarrow G^{\prime}$ be a homomorphism of affine group schemes over $k$ and let

$$
\widetilde{\theta}: \operatorname{Rep}_{k}\left(G^{\prime}\right) \longrightarrow \operatorname{Rep}_{k}(G)
$$

be the functor given by sending $\rho^{\prime}: G^{\prime} \rightarrow \mathrm{GL}(V)$ to $\rho^{\prime} \circ \theta: G \rightarrow \mathrm{GL}(V)$. An object $\rho: G \rightarrow \mathrm{GL}(V)$ in $\operatorname{Rep}_{k}(G)$ is said to be a subquotient of an object $\eta: G \rightarrow \mathrm{GL}(W)$ in $\operatorname{Rep}_{k}(G)$ if there are two $G$-submodules $V_{1} \subset V_{2}$ of $W$ such that $V \cong V_{2} / V_{1}$ as $G$-modules.

Proposition 5.2.2 (Proposition 2.21, $[\mathrm{DM}]$ ). Let $\theta: G \longrightarrow G^{\prime}$ be a homomorphism of affine algebraic groups over $k$. Then

(a) $\theta$ is faithfully flat if and only if the functor $\widetilde{\theta}$ in (5.2.1) is fully faithful and given any subobject $W \subset \widetilde{\theta}\left(V^{\prime}\right)$, with $V^{\prime} \in \operatorname{Rep}_{k}\left(G^{\prime}\right)$, there is a subobject $W^{\prime} \subset V^{\prime}$ in $\operatorname{Rep}_{k}\left(G^{\prime}\right)$ such that $\widetilde{\theta}\left(W^{\prime}\right) \cong W$ in $\operatorname{Rep}_{k}(G)$.

(b) $f$ is a closed immersion if and only if every object of $\operatorname{Rep}_{k}(G)$ is isomorphic to a subquotient of an object of the form $\tilde{\theta}\left(V^{\prime}\right)$, for some $V^{\prime} \in$ $\operatorname{Rep}_{k}\left(G^{\prime}\right)$. 
Proposition 5.2.3. The homomorphism

$$
\tilde{f}: \pi^{S}(X, x)_{\mathrm{ab}} \rightarrow \pi^{S}\left(\mathcal{H} i l b_{X}^{n}, \widetilde{n x}\right)
$$

defined in (4.3.2) is faithfully flat.

Proof. We will use Proposition 5.2.2 (a). Let $E_{1}$ be an object in the category $\operatorname{Vect}_{0}^{s}\left(\mathcal{H} i l b_{X}^{n}\right)=\mathcal{C}^{\text {nf }}\left(\mathcal{H} i l b_{X}^{n}\right)$. Let $\mathcal{G}_{1}:=\mathscr{G}\left(E_{1}\right)$ be the vector bundle as defined in (4.2.11). Clearly $\mathcal{G}_{1}$ has the same rank as that of $E_{1}$. If $\mathcal{G}_{2} \subset \mathcal{G}_{1}$ is a subbundle corresponding to a representation of $\pi^{S}(X, x)_{\mathrm{ab}}$, we need to show that there is a subbundle $E_{2} \subset E_{1}$ such that $\mathcal{G}_{2}=\mathscr{G}\left(E_{2}\right)$. We will prove this by induction on the rank of $E_{1}$. If $\operatorname{rank}\left(E_{1}\right)=1$, there is nothing to prove. Assume that $\operatorname{rank}\left(E_{1}\right) \geq 2$.

The vector bundles $\mathcal{G}_{i}$ correspond to representations

$$
\pi^{S}\left(X^{n},(x, \ldots, x)\right) \stackrel{f_{0}}{\longrightarrow} \pi^{S}(X, x)_{\mathrm{ab}} \stackrel{\rho_{i}}{\longrightarrow} \mathrm{GL}\left(V_{i}\right) .
$$

Since $\pi^{S}(X, x)_{\text {ab }}$ is an abelian affine $k$-group scheme, it follows from [Wat79, Theorem 9.4 , p. 70] that every irreducible representation of it is one dimensional. From this one easily checks that the $\pi^{S}(X, x)_{\text {ab-module }} V_{1} / V_{2}$ will have a one dimensional quotient. Thus, there is a one dimensional quotient $V_{1} \rightarrow L_{1}$ such that $V_{2}$ is a $\pi^{S}(X, x)_{\mathrm{ab}}$-submodule of the kernel of this homomorphism. Let $\mathcal{L}$ be the line bundle on $X^{n}$ corresponding to the representation $L_{1}$. Then it is clear that $\mathcal{L}$ is $S_{n}$-invariant (see Corollary 4.1.3) and there is an $S_{n}$-equivariant exact sequence of bundles

$$
0 \longrightarrow \mathcal{K} \longrightarrow \mathcal{G}_{1} \longrightarrow \mathcal{L} \longrightarrow 0
$$

on $X^{n}$ such that $\mathcal{G}_{2} \subset \mathcal{K}$.

It follows from Proposition 5.1.1 that $L^{\prime}:=\left(\psi_{*} \mathcal{L}\right)^{S_{n}}$ is a locally free line bundle on all of $S^{n}(X)$ and satisfies $\psi^{*} L^{\prime}=\mathcal{L}$. Let $L:=\varphi^{*} L^{\prime}$, then it is easy to check that $L$ is numerically flat on $\mathcal{H} i l b_{X}^{n}$.

We claim that the following complex of sheaves on $W$

$$
\left.\left.\left.0 \rightarrow\left(\psi_{*} \mathcal{K}\right)^{S_{n}}\right|_{W} \rightarrow\left(\psi_{*} \mathcal{G}_{1}\right)^{S_{n}}\right|_{W} \rightarrow\left(\psi_{*} \mathcal{L}\right)^{S_{n}}\right|_{W} \rightarrow 0
$$

is exact. The sequence (5.2.4) can fail to be exact only on the right. Note that $\psi_{*}\left(\mathcal{G}_{1}\right)^{S_{n}}$ restricted to $W$ is $\varphi_{*}\left(\left.E_{1}\right|_{V}\right)$. Let $J$ be the cokernel:

$$
\left.\varphi_{*}\left(\left.E_{1}\right|_{V}\right) \rightarrow L^{\prime}\right|_{W} \rightarrow J \rightarrow 0 .
$$

Pulling this back by $\psi$ we get the following commutative diagram on $\psi^{-1}(W)$ with exact rows.

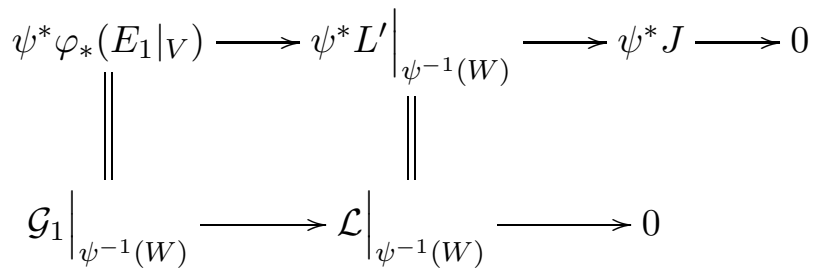


This shows that $\psi^{*} J=0$. It is easy to conclude that $J=0$, since $\psi$ is surjective. This proves the exactness of (5.2.4). It follows that $K^{\prime}:=$ $\left(\psi_{*} \mathcal{K}\right)^{S_{n}}$ is locally free on $W$. Applying $\varphi^{*}$ to (5.2.4), we get the following short exact sequence of locally free sheaves on $V$.

$$
\left.\left.\left.0 \longrightarrow\left(\varphi^{*} K^{\prime}\right)\right|_{V} \longrightarrow E_{1}\right|_{V} \longrightarrow L\right|_{V} \longrightarrow 0 .
$$

Since both $E_{1}$ and $L$ are locally free on a smooth variety and $\mathcal{H} i l b_{X}^{n} \backslash V$ has codimension $\geq 2$, it follows that this morphism on $V$ extends to a morphism $E_{1} \rightarrow L$. This being a nonzero morphism of numerically flat vector bundles and $L$ being of rank one, it follows that $E_{1} \rightarrow L$ is surjective.

It is clear that on $X^{n}$ we have $\mathscr{G}(L)=\mathcal{L}$. Let $K$ denote the kernel of the homomorphism $E_{1} \longrightarrow L$. It is clear that $\mathscr{G}(K)=\mathcal{K}$. Since $\mathcal{G}_{2} \subset \mathcal{K}$ the assertion that there is $E_{2} \subset E_{1}$ such that $\mathcal{G}_{2}=\mathscr{G}\left(E_{2}\right)$ follows by induction on rank.

To complete the proof of the proposition we need to show that if $E_{1}$ and $E_{2}$ are numerically flat vector bundles on $\mathcal{H} i b_{X}^{n}$ then the natural map

$$
\operatorname{Hom}_{\mathcal{H} i l b_{X}^{n}}\left(E_{1}, E_{2}\right) \longrightarrow \operatorname{Hom}_{X^{n}}\left(\mathcal{G}_{1}, \mathcal{G}_{2}\right)
$$

is bijective. It is clear that this natural map is injective (faithful). Therefore, it suffices to show the following. If $\mathcal{G}=\mathscr{G}(E)$, where $E$ is a numerically flat vector bundle on $\mathcal{H} i l b_{X}^{n}$, then any nonzero homomorphism $\phi: \mathcal{O}_{X^{n}} \longrightarrow \mathcal{G}$ comes from a nonzero homomorphism $\widetilde{\phi}: \mathcal{O}_{\mathcal{H}} i b_{X}^{n} \longrightarrow E$. Since the homomorphism $\pi^{S}\left(X^{n}, x\right) \longrightarrow \pi^{S}(X, x)_{\mathrm{ab}}$ is faithfully flat, and $\mathcal{G}$ arises from a representation of $\pi^{S}(X, x)_{\mathrm{ab}}$, it follows that $\phi$ is a map between two representations of $\pi^{S}(X, x)_{\mathrm{ab}}$. This shows that $\phi$ is $S_{n}$-equivariant on $X^{n}$. Now from the preceding discussion it follows that $\phi$ arises from a morphism $\mathcal{O}_{\mathcal{H} i l b_{X}^{n}} \longrightarrow E$.

5.3. Closed immersion. In this subsection we show that the homomorphism $\tilde{f}$ in (4.3.2) is a closed immersion. For this, we will apply 5.2.2 (b).

Let $q \in S^{n}(X)$ be a point of type $\left\langle n_{1}, n_{2}, \ldots, n_{r}\right\rangle$. Let $\tilde{q}_{i}$, for $i=$ $1,2, \ldots, m$, denote the points in the fiber $\psi^{-1}(q)$. The stabilizer of $\tilde{q}_{i}$, denoted $\operatorname{St}\left(\tilde{q}_{i}\right)$, is isomorphic to $S_{n_{1}} \times S_{n_{2}} \times \ldots \times S_{n_{r}}$. Let $A$ denote the local ring $\mathcal{O}_{S^{n}(X), q}$ and let $B$ denote the semilocal ring $\mathcal{O}_{X^{n}} \otimes_{\mathcal{O}_{S^{n}(X)}} A$. Then $B$ is a finite $A$ module and $A=B^{S_{n}}$.

Let $M$ be a $B$-module such that the action of $S_{n}$ on $B$ lifts to an action of $S_{n}$ on $M$. There is a short exact sequence of $A$ modules

$$
0 \rightarrow M^{S_{n}} \rightarrow M \rightarrow \bigoplus_{g \in S_{n}} M
$$

where the last map is given by $m \mapsto(g \cdot m-m)_{g \in S_{n}}$. Let $\widehat{A}$ be the completion of $A$ with respect to its maximal ideal. Applying the functor $-\otimes_{A} \widehat{A}$, we 
conclude that the following natural map is an isomorphism.

$$
\widehat{M^{S_{n}}} \stackrel{\sim}{\longrightarrow} \widehat{M}^{S_{n}}
$$

The ring $\widehat{B}=B \otimes_{A} \widehat{A}$ decomposes as

$$
\widehat{B} \cong \bigoplus_{i=1}^{m} \widehat{B}_{i},
$$

where $\widehat{B}_{i}$ denotes the completion of $B$ at the maximal ideal corresponding to the point $\tilde{q}_{i}$, for all $i=1, \ldots, m$. Applying the functor $M \otimes_{B}-$ to the above isomorphism (5.3.1) we see that

$$
\widehat{M} \cong \bigoplus_{i=1}^{m} \widehat{M}_{i},
$$

where $M_{i}$ is the localization of $M$ at the maximal ideal corresponding to the point $\tilde{q}_{i}$. Taking $S_{n}$-invariants in (5.3.2), it easily follows that

$$
\widehat{M}^{S_{n}} \cong \widehat{M}_{i}^{\mathrm{St}\left(\tilde{q}_{i}\right)}, \quad \forall i
$$

Proposition 5.3.3. With notation as above, whenever $\operatorname{char}(k)>n_{1}$, any $S_{n}$-equivariant surjective $B$-module homomorphism $f: M \longrightarrow N$ of finitely generated B-modules descends to surjective A-module homomorphism of their $S_{n}$-invariants $M^{S_{n}} \longrightarrow N^{S_{n}}$.

Proof. Suppose we have an $S_{n}$-equivariant exact sequence of $B$-modules

$$
M \longrightarrow N \longrightarrow 0 \text {. }
$$

Taking $S_{n}$-invariants we get a homomorphism of $A$-modules

$$
M^{S_{n}} \longrightarrow N^{S_{n}} \text {. }
$$

To check this is surjective, it suffices to check that the map (5.3.4) is surjective after passing to the completion. From the preceding discussion, it follows that it suffices to check that

$$
\widehat{M}_{i}^{\mathrm{St}\left(\tilde{q}_{i}\right)} \longrightarrow \widehat{N}_{i}^{\mathrm{St}\left(\tilde{q}_{i}\right)}
$$

is surjective for one (and hence any) $i$. We know that $\widehat{M} \rightarrow \widehat{N}$ is surjective. Thus, the above map in (5.3.5) will be surjective if we can lift a section of $\widehat{N}_{i}^{\mathrm{St}\left(\tilde{q}_{i}\right)}$ to $\widehat{M}$ and average it, that is, apply the operator

$$
\frac{1}{\# \operatorname{St}\left(\tilde{q}_{i}\right)} \sum_{g \in \operatorname{St}\left(\tilde{q}_{i}\right)} g .
$$

This is possible if $\operatorname{char}(k)=p>n_{1}$ (c.f. inequalities (3.2.1)).

Proposition 5.3.6. Let $\mathcal{G}$ be a numerically flat $S_{n}$-invariant locally free sheaf on $X^{n}$.

(i) Let $q \in S^{n}(X)$ be a point of type $\left\langle n_{1}, n_{2}, \ldots, n_{r}\right\rangle$. Assume that $\operatorname{char}(k)=$ $p>n_{1}$. Then the sheaf $\left(\psi_{*} \mathcal{G}\right)^{S_{n}}$ is locally free in a neighborhood of $q$. 
(ii) Let $U_{0}$ denote the largest open subset where $\left(\psi_{*} \mathcal{G}\right)^{S_{n}}$ is locally free. Then on $\psi^{-1}\left(U_{0}\right)$ the natural homomorphism

$$
\psi^{*}\left(\left(\psi_{*} \mathcal{G}\right)^{S_{n}}\right) \longrightarrow \mathcal{G}
$$

is an isomorphism.

Proof. If $\mathcal{G}$ has rank 1 then $\left(\psi_{*} \mathcal{G}\right)^{S_{n}}$ is locally free on all of $S^{n}(X)$ and of rank one, see Proposition 5.1.1. Since $\mathcal{G}$ corresponds to a representation of an abelian group scheme, it follows that there is an $S_{n}$-equivariant exact sequence of locally free sheaves on $X^{n}$

$$
0 \longrightarrow \mathcal{K} \longrightarrow \mathcal{G} \longrightarrow \mathcal{L} \longrightarrow 0,
$$

with $\operatorname{rank}(\mathcal{L})=1$. By induction on rank of $\mathcal{G}$, it suffices to show that the homomorphism on the right of the following exact sequence

$$
0 \longrightarrow\left(\psi_{*} \mathcal{K}\right)^{S_{n}} \longrightarrow\left(\psi_{*} \mathcal{G}\right)^{S_{n}} \longrightarrow\left(\psi_{*} \mathcal{L}\right)^{S_{n}}
$$

is surjective in a neighbourhood of $q$. This surjection can be checked after passing to a formal neighbourhood of $q$. Now the first assertion of the Proposition follows from the above Proposition 5.3.3.

To prove the second assertion, note that both sheaves are locally free of the same rank over $\psi^{-1}\left(U_{0}\right)$. The locus where the natural homomorphism (5.3.7) is not an isomorphism is either empty or a closed subset of codimension 1 in $\psi^{-1}\left(U_{0}\right)$. However, we know that the morphism $\psi$ is finite étale over the smooth locus of $S^{n}(X)$, hence the homomorphism (5.3.7) is an isomorphism on the inverse image of the smooth locus of $S^{n}(X)$. Since the complement of the smooth locus of $S^{n}(X)$ has codimension 2 , it follows that the natural map in (5.3.7) is an isomorphism over $\psi^{-1}\left(U_{0}\right)$.

Lemma 5.3.8. Let $T \subset S^{n}(X)$ be open. If $\delta: E_{1} \rightarrow E_{2}$ is a morphism between locally free sheaves on $T$, such that $\psi^{*} \delta$ is an isomorphism on $\psi^{-1}(T)$, then $\delta$ is an isomorphism.

Proof. For a locally free sheaf $E$ on $T$, we have $E \cong\left[\psi_{*}\left(\psi^{*} E\right)\right]^{S_{n}}$. Thus, if $\delta: E_{1} \rightarrow E_{2}$ is a morphism on $T$, such that $\psi^{*} \delta$ is an isomorphism on $\psi^{-1}(T)$, then taking pushforward and $S_{n}$ invariants, it follows that $\delta$ is an isomorphism.

Proposition 5.3.9. Let $\operatorname{char}(k)>3$. Then the homomorphism $\tilde{f}$ in (4.3.2) is a closed immersion.

Proof. By Proposition 5.2.2 (b) it suffices to show that every $S_{n}$-invariant numerically flat bundle $\mathcal{G}$ on $X^{n}$ arises in the way described in Proposition 4.2.10. In other words, we have to show that there is a numerically flat bundle $E$ on $\mathcal{H} i l b_{X}^{n}$ such that $\mathcal{G}=\left(j_{*}\left(\psi^{*} \varphi_{*}\left(\left.E\right|_{V}\right)\right)\right)^{\vee \vee}$.

Let $T \supset W$ be the open subset of $S^{n}(X)$ containing $W$ and points of type $\langle 3,1,1, \ldots, 1\rangle$ and $\langle 2,2,1, \ldots, 1\rangle$. Then $\varphi^{-1}(T)$ is an open subset of 
$\mathcal{H} i l b_{X}^{n}$ such that $\mathcal{H} i l b_{X}^{n} \backslash \varphi^{-1}(T)$ has codimension at least 3 in $\mathcal{H} i l b_{X}^{n}$. Let $i: \varphi^{-1}(T) \hookrightarrow \mathcal{H} i l b_{X}^{n}$ denote the inclusion. Define

$$
E:=\left(i_{*} \varphi^{*}\left(\left(\left.\left(\psi_{*} \mathcal{G}\right)\right|_{T}\right)^{S_{n}}\right)\right)^{\vee \vee} .
$$

By Proposition 5.3.6 we see that $\left(\psi_{*} \mathcal{G}\right)^{S_{n}}$ is locally free on $T$ and on $\psi^{-1}(T)$ the natural homomorphism $\psi^{*}\left(\left(\psi_{*} \mathcal{G}\right)^{S_{n}}\right) \rightarrow \mathcal{G}$ is an isomorphism. Consider the natural homomorphisms

$$
F^{*}\left(\left(\psi_{*} \mathcal{G}\right)^{S_{n}}\right) \longrightarrow\left(F^{*} \psi_{*}(\mathcal{G})\right)^{S_{n}} \longrightarrow\left(\psi_{*}\left(F^{*} \mathcal{G}\right)\right)^{S_{n}},
$$

where $F$ denotes the absolute Frobenius morphism. We claim that the above composite homomorphism is an isomorphism over T. Applying $\psi^{*}$ to the above exact sequence, we get the following commutative diagram.

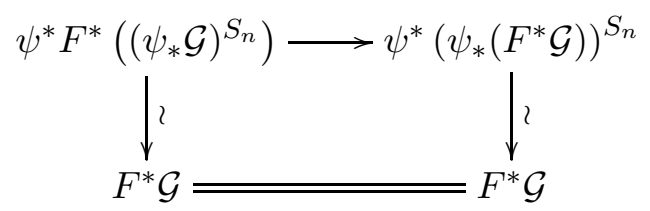

The two vertical arrows are isomorphisms on $T$ because of Proposition 5.3.6. It follows from Lemma 5.3.8 that the composite homomorphism in (5.3.10) is an isomorphism over $T$. It follows that $F^{*} E \cong\left(i_{*} \varphi^{*}\left(\psi_{*}\left(\left.F^{*} \mathcal{G}\right|_{T}\right)^{S_{n}}\right)\right)^{\vee \vee}$. Now imitating the proof of Proposition 4.2.10 we see that $E$ is locally free and numerically flat on $\mathcal{H} i l b_{X}^{n}$. It is clear that $\mathscr{G}(E)=\mathcal{G}$ (see the construction in the proof of Proposition 4.2.10). This proves the Proposition.

Theorem 5.3.11. Let $\operatorname{char}(k)>3$. Then the homomorphism

$$
\widetilde{f}: \pi^{S}(X, x)_{\mathrm{ab}} \longrightarrow \pi^{S}\left(\mathcal{H} i l b_{X}^{n}, \widetilde{n x}\right)
$$

in (4.3.2) is an isomorphism.

Proof. Since $\tilde{f}$ is faithfully flat by Proposition 5.2.3 and closed immersion by Proposition 5.3.9, it is an isomorphism.

From the above theorem we may easily conclude the following.

Theorem 5.3.12. Let $\operatorname{char}(k)>3$. There is an isomorphism of affine $k$-group schemes

$$
\tilde{f}^{?}: \pi^{?}(X, x)_{\mathrm{ab}} \longrightarrow \pi^{?}\left(\mathcal{H} i l b_{X}^{n}, \widetilde{n x}\right)
$$

where $?=N$, ét.

Let $E$ be an essentially finite vector bundle over a connected, reduced and proper $k$-scheme $X$. Then there is a finite $k$-group scheme $G$, a principal $G$ bundle $p: P \rightarrow X$ and a finite dimensional $k$-linear representation $\rho: G \rightarrow$ $\operatorname{GL}\left(V^{\prime}\right)$ such that $E$ is the vector bundle associated to the representation $\rho$. It follows from the proof of [Nor76, Proposition 3.8] that there is a finite vector bundle $\mathcal{V}$ on $X$ such that $E$ is a subbundle of $\mathcal{V}$. It is clear that the functor $\mathscr{G}$ in Proposition 4.2.10 takes a finite vector bundle to a finite vector 
bundle. It easily follows that $\mathscr{G}$ takes essentially finite vector bundles to essentially finite vector bundles. With these remarks we leave the details of the proof of Theorem 5.3.12 to the reader.

\section{Declaration of competing interest.}

The authors declare that they have no conflict of interest.

\section{REFERENCES}

[Bea83] Arnaud Beauville. Variétés Kähleriennes dont la première classe de Chern est nulle. J. Differential Geom., 18(4):755-782 (1984), 1983. URL http://projecteuclid.org/euclid.jdg/1214438181.

[BH15] Indranil Biswas and Amit Hogadi. On the fundamental group of a variety with quotient singularities. Int. Math. Res. Not. IMRN, (5):1421-1444, 2015. doi:10.1093/imrn/rnt261.

[BPS06] Indranil Biswas, A. J. Parameswaran, and S. Subramanian. Monodromy group for a strongly semistable principal bundle over a curve. Duke Math. J., 132(1):1-48, 2006. doi:10.1215/S0012-7094-06-13211-8.

[DM] Pierre Deligne and James S. Milne. Tannakian Categories. Chapter 2, pages 101-228. In: Hodge Cycles, Motives, and Shimura Varieties. Lecture Notes in Mathematics, vol 900. Springer-Verlag, Berlin-New York, 1982. doi:10.1007/978-3-540-38955-2_4.

[EM11] Hélène Esnault and Vikram B. Mehta. Weak density of the fundamental group scheme. Int. Math. Res. Not. IMRN, (13):3071-3081, 2011. doi:10.1093/imrn/rnq187.

$\left[\mathrm{FGI}^{+} 05\right]$ Barbara Fantechi, Lothar Göttsche, Luc Illusie, Steven L. Kleiman, Nitin Nitsure, and Angelo Vistoli. Fundamental algebraic geometry, volume 123 of Mathematical Surveys and Monographs. American Mathematical Society, Providence, RI, 2005. Grothendieck's FGA explained. doi:10.1090/surv/123.

[Fog73] John Fogarty. Algebraic families on an algebraic surface. II. The Picard scheme of the punctual Hilbert scheme. Amer. J. Math., 95:660-687, 1973. doi:10.2307/2373734.

[Fog77] John Fogarty. Line bundles on quasi-symmetric powers of varieties. J. Algebra, 44(1):169-180, 1977. doi:10.1016/0021-8693(77)90171-5.

[Har77] Robin Hartshorne. Algebraic geometry. Springer-Verlag, New York-Heidelberg, 1977. Graduate Texts in Mathematics, No. 52. doi:10.1007/978-1-4757-3849-0.

[Har80] Robin Hartshorne. Stable reflexive sheaves. Math. Ann., 254(2):121-176, 1980. doi:10.1007/BF01467074.

[Iar72] Anthony Iarrobino. Punctual Hilbert schemes. Bull. Amer. Math. Soc., 78:819823, 1972. doi:10.1090/S0002-9904-1972-13049-0.

[Lan04] Adrian Langer. Semistable sheaves in positive characteristic. Ann. of Math. (2), 159(1):251-276, 2004. doi:10.4007/annals.2004.159.251.

[Lan11] Adrian Langer. On the S-fundamental group scheme. Ann. Inst. Fourier (Grenoble), 61(5):2077-2119 (2012), 2011. doi:10.5802/aif.2667.

[Lan12] Adrian Langer. On the S-fundamental group scheme. II. J. Inst. Math. Jussieu, 11(4):835-854, 2012. doi:10.1017/S1474748012000011.

[Nor76] Madhav V. Nori. On the representations of the fundamental group. Compositio Math., 33(1):29-41, 1976. URL http: //www . numdam. org/item?id=CM_1976__33_1_29_0. 
[Nor82] Madhav V. Nori. The fundamental group-scheme. Proc. Indian Acad. Sci. Math. Sci., 91(2):73-122, 1982. doi:10.1007/BF02967978.

[Stk] The Stack Project. https://stacks.math.columbia.edu.

[Wat79] William C. Waterhouse. Introduction to affine group schemes, volume 66 of Graduate Texts in Mathematics. Springer-Verlag, New York-Berlin, 1979. doi:10.1007/978-1-4612-6217-6.

(A. Paul) Department of Mathematics, Indian Institute of Technology BomBay, Powai, Mumbai 400076, Maharashtra, India.

E-mail address: arjun.math.tifr@gmail.com

(R. Sebastian) Department of Mathematics, Indian Institute of Technology Bombay, Powai, Mumbai 400076, Maharashtra, India.

E-mail address: ronnie@math.iitb.ac.in 